\title{
RX-207, Small Molecule Inhibitor Of Protein Interaction With Glycosaminoglycans (SMIGs), Reduces Experimentally Induced Inflammation And Increases Survival Rate In Cecal Ligation And Puncture (CLP) Induced Sepsis
}

\author{
Stefan Juhas ${ }^{c, 1}$, Nicholas Harris ${ }^{a, b}{ }^{*}$, Gabriela II'kovaa,2, Pavol Rehák ${ }^{c}$ Ferenc Zsilad, Faina \\ Yurgenzon Kogan ${ }^{a}$, Orly Lahmy ${ }^{a}$, Regina Zhuk ${ }^{a}$, Paul Gregor ${ }^{a, e},{ }^{*}$ and Juraj Koppel ${ }^{c}$
}

aRimonyx Pharmaceuticals Ltd., Rabin Science Park, Ness-Ziona 70400, Israel ${ }^{b}$ Ephraim Katzir Department of Biotechnology Engineering, ORT Braude Academic College of Engineering, Karmiel, Israel Institute of Animal Physiology, Slovak Academy of Sciences, 04001 Kosice, Slovakia dBiomolecular Self-Assembly Group, Institute of Materials and Environmental Chemistry, Research Centre for Natural Sciences, Budapest, Hungary 'GISMO Therapeutics Inc., Lexington, KY 40506, USA.

${ }^{1}$ Present address: GYN-FIV a.s., Záhradnícka 42, 821085 Bratislava, Slovakia.

2Present address: Institute of Animal Physiology and Genetics of the ASCR, v. v. i., Rumburská 89, 27721 Liběchov, Czech Republic

Running Title Small molecule inhibitors of deleterious inflammation and sepsis

\section{*Corresponding authors:}

Dr. Nicholas Harris

Ephraim Katzir Department of Biotechnology Engineering

ORT Braude Academic College of Engineering

Snonit 51,

Karmiel 2161002

Israel

(Tel) 97249086472, (Fx) 97249901839, (Email) nharris@braude.ac.il

Dr. Paul Gregor

GISMO Therapeutics Inc., A253 ASTECC-UK

Lexington

KY 40506, USA

(Tel) 13472517342, (Email) paul@gismotherapeutics.com

\section{Acknowledgement}

This study was supported by the Project No. ITMS 26220120066, of the Research and Development Operational Program funded by the European Regional Development Fund 


\begin{abstract}
The fused quinazolinone derivative, RX-207, is chemically and functionally related to small molecule inhibitors of protein binding to glycosaminoglycans (SMIGs). Composed of a planar aromatic amine scaffold, it inhibits protein binding to glycosaminoglycans (GAGs). RX-207 reduced neutrophil migration in thioglycollate induced peritonitis (37\%), inhibited carrageenan induced paw edema (32\%) and cerulein induced pancreatitis (28\%) and increased animal survival in the mouse model of cecal ligation and puncture (CLP) induced sepsis (60\%). The mechanism of RX-207 action, analyzed by UV spectroscopy, confirmed that which was elucidated for chemically related anti-inflammatory SMIGs. RX-207 binding to cell surface GAGs can account for the inhibition of neutrophil recruitment via the microvasculature and as a consequence the reduction of neutrophil mediated tissue damage in the animal models of inflammation and improved survival of mice in CLP induced sepsis.
\end{abstract}

Keywords: heparin binding protein; glycosaminoglycan; neutrophil; inflammation; sepsis 


\section{Introduction}

Contemporary medicine treats inflammatory disorders with a variety of medications. Corticosteroids are the most effective drugs for acute inflammation and are efficacious, at a low dose, in treating chronic inflammation [1]. Inflammatory disorders that are refractory to treatment, such as acute pancreatitis and sepsis, can be fatal. The absence of effective treatment of sepsis is not for want of effort, or resource. There are more than four hundred reports of preclinical studies of the pro-inflammatory cytokine, tumor necrosis factor alfa (TNF- $\alpha$ ), alone and more than one hundred clinical trials have been carried out on antiinflammatory drug candidates [2]. Many and various explanations have been given for the clinical failure of drugs in treating sepsis [3]. One, for example, is the difficulty in defining the disease [4], another is the temporal heterogeneity in sepsis development and a third explanation is the biological heterogeneity resulting from patient age, comorbidities and pathogens [5]. What is true for sepsis and inflammatory disorders in general, is that inflammation is the net result of the activities of multiple biochemical pathways. Modulation of any one pathway, therefore, is unlikely to influence the course of the illness in a significant way. Profound changes in the endothelium of venules, as a result of sepsis, cause vasodilation, loss of barrier function and widespread tissue edema [6]. Neutrophils, the principal effectors of endothelial damage in sepsis [7], are potential targets for antiinflammatory drugs. Therapeutic modulation of inflammatory neutrophils potentially could reduce tissue edema and prevent the damage caused by the reactive oxygen species, proteases and pro-inflammatory cytokines that they secrete [8]. Anti-inflammatory small molecule inhibitors of protein binding to glycosaminoglycans (SMIGs) have been described [9] and their mechanism of action in experimental models of inflammation elucidated [10]. The current manuscript describes the anti-inflammatory activity of a novel SMIG, 
demonstrates its efficacy in experimental sepsis and delineates and confirms the mechanism of action that can account for the inhibition of inflammatory neutrophil adhesion and activity in the disease. 


\title{
Materials and Methods
}

\author{
Materials \\ Quinazolinone derivatives were purchased from, or custom-synthesized by Life Chemicals \\ Ltd. (Kiev, Ukraine) and ChemDiv (San Diego, CA); heparin sodium salt (porcine intestinal \\ mucosa, Cat\# 51551), chondroitin 6-sulfate sodium salt (shark cartilage, Cat\# C4384), \\ carrageenan (Cat\# C-3889), cerulein (Cat\# C-9026), Tween 80, DMSO, thioglycollate (Cat\# \\ T-0632) were purchased from Sigma-Aldrich Corporation, (St Louis, MO); Türk's reagent was \\ purchased from (Merck, Germany, Cat\# 109277); Balb/c mice were provided by Velaz \\ (Prague, Czech Republic); the solubility of RX-207 is $20 \mathrm{mg} / \mathrm{mL}$ in $50 \mathrm{mM}$ citric acid.
}

\section{Methods}

\section{Thioglycollate-induced peritonitis ${ }^{1}$}

$\mathrm{BALB} / \mathrm{c}$ mice (6 weeks old, $\sim 19 \mathrm{~g}$ in weight, 12 mice/group) received p.o. inhibitor compound in $0.1 \mathrm{~mL}$ methylcellulose 1 hour before administration of thioglycollate. Control groups received vehicle only. Mice were injected intraperitoneally with $1 \mathrm{~mL}$ of $3 \%$ thioglycollate broth [11]. Mice were sacrificed after 3 hours, and the peritoneal cavities were lavaged with $5 \mathrm{~mL}$ of ice-cold saline containing $2 \mathrm{mM}$ EDTA to prevent clotting. After red blood cell lysis, leukocytes were counted in a hemocytometer. Neutrophils were counted after staining with Türk's reagent. Data was expressed as mean \pm SEM, and statistical analysis was performed by Student $t$ test.

\section{Quinazolinone (RX-207) formulation}

RX 207 was dissolved in a mixture of propylenglycol (41\%, Fluka, Biochemika), PEG400 (9\%, Fluka, Biochemika) and water (pharmaceutical grade). 


\section{Carrageenan-induced paw edema ${ }^{1}$}

Acute edema was induced in the right hind paw of BALB/C mice ( 8 weeks old, $\sim 21 \mathrm{~g}$ in weight, 12 mice/group) by injecting $0.05 \mathrm{~mL}$ of freshly prepared solution of $1 \%$ carrageenan after $60 \mathrm{~min}$ of test compound administration [12]. The left paw received $0.05 \mathrm{~mL}$ of saline, which served as a control. Carrageenan was injected under the plantar region of right hind paw and the paw thickness was measured at 4 hours after carrageenan challenge using a Mitutoyo engineer's micrometer expressed as the difference between right and left pad as mean \pm SEM. Results were evaluated by Student's $t$ test.

\section{Acute pancreatitis ${ }^{1}$}

Acute pancreatitis was induced in Balb/c mice ( 8 weeks old, $\sim 22 \mathrm{~g}$ in weight, 10-11 mice per group) by injecting cerulein (50 $\mu \mathrm{g} / \mathrm{kg}$, i.p., X7; [13]). The cerulein model of acute pancreatitis is a commonly used pharmaceutical industry model. Test compounds were administered i.m or i.v. 1h prior to first cerulein injection. One hour after the last cerulein injection blood samples were taken. Serum amylase was analysed by standard methods [14]. Amylase data were calculated as units per volume and expressed as mean \pm SEM. Results were evaluated by Student's $t$ test.

\section{Cecal ligation and puncture (CLP, a model of sepsis) ${ }^{1}$}

CLP was performed in the control and experimental Balb/c mice ( 8 weeks old, $\sim 22 \mathrm{~g}$ in weight, 9-10 mice per group) as described by Fink [15]. The experimental mice were given RX-207 or RX-205 (20 mg/kg, 0.1ml, i.m.), 24 hours before surgery and once per day for the duration of the study. Survival data were evaluated by Log-rank (Mantel-Cox) test.

\section{UV absorption spectroscopy}

UV absorption spectra were recorded on a JASCO J-715 spectropolarimeter at $25 \pm 0.2{ }^{\circ} \mathrm{C}$. Temperature control was provided by a Peltier thermostat equipped with magnetic stirring. Each spectrum represents the average of two scans obtained by collecting data at a scan 
speed of $200 \mathrm{~nm} / \mathrm{min} .2 .3 \mathrm{mM}$ stock solution of RX-207 was prepared using ultra-pure water and a small volume of ethanol. 25 or $30 \mu \mathrm{L}$ stock solution of the ligand was added to $1.8 \mathrm{~mL}$ ultra-pure water in a rectangular quartz cell of $1 \mathrm{~cm}$ optical path length (Hellma, USA). Drug samples obtained by this way were subsequently titrated by small aliquots of concentrated aqueous solution of heparin and chondroitin 6-sulfate. Due to their poly-disperse nature, glycosaminoglycan (GAG) concentrations were expressed using the molecular weight of the average repeating disaccharide units: 665 and $503 \mathrm{~g} / \mathrm{mol}$ for heparin and chondroitin 6sulfate, respectively. Absorption curves of drug-GAG mixtures were corrected by subtracting the spectra of blank water solution.

${ }^{1}$ The animal experiments were reviewed and approved by the Ethical Committee for animal experimentation of the Institute of Animal Physiology, approved by the State Veterinary and Food Administration of the Slovak Republic and were performed in accordance with Slovak legislation based on EC Directive 86/609/EEC on the protection of animals used for experimental and other scientific purposes. 


\section{Results}

\section{RX-207 and RX-205, inhibitors of neutrophil migration}

An in vitro screen of SMIGs identified the anti-inflammatory quinazolinone, $R X-211$, with $I_{50}$ values in the low micro-molar range and broad activity against inflammatory chemokines and cytokines [9]. Derivatives of related compounds, the fused quinazolinones, RX-207 (azepino[2,1-b]quinazoline-6,7,8,9,10,12-hexahydro-12-oxo- $N$-(1-methylethyl-piperidinyl-4)3-carboxamide, Figure 1A and RX-205 (pyrrolo[2,1-b]quinazoline-1,2,3,9-tetrahydro-9-oxo$N$-(1-methyl-piperidinyl-4)-6-carboxamide), Figure 1B, were predicted to have antiinflammatory activity in animal models of inflammation.

Thioglycollate induced peritonitis that results in a massive neutrophil infiltrate into the peritoneum [16], is a frequently used animal model to study acute inflammation [17]. Oral administration of RX-205 and RX-207 (50 mg/kg) inhibited neutrophil migration into the peritoneal cavity by $38 \%$ and $37 \%$, respectively (Figure 2 ).

\section{RX-207 anti-inflammatory activity}

Carrageenan-induced inflammation is characterized by the migration of leukocytes, mainly neutrophils, to the site of inflammation [18]. Carrageenan-induced edema of the mouse paw is a model of acute local inflammation to evaluate the anti-inflammatory activity of investigational compounds [12]. Intra-muscular (i.m.) injection of RX-207 (1 mg/kg, 0.1ml) inhibited carrageenan-induced paw edema in mice (32\%), as measured by a reduction in paw thickness (Figure 3). RX-205 (25 mg/kg), administered i.v., reduced paw thickness by $24 \%(p<0.001$, data not shown).

Supra-physiologic doses of cerulein cause acute pancreatitis in mice reproducing morphological and biochemical features of the human disease [13]. Models of cerulein 
induced pancreatitis, characterized by increased serum amylase activity, have been used to study acute pancreatitis and to test potential drugs [19]. RX-207 (25 mg/kg, 0.1ml), administered i.m., reduced serum levels of amylase by $28 \%$ (Figure 4). RX-205 (20 mg/kg), administered i.v., reduced amylase activity by $34 \%$ ( $p<0.01$, data not shown).

\section{RX-207 and RX-205 therapeutic activity}

Cecal Ligation and Puncture (CLP) causes poly-microbial sepsis, a procedure used for modeling sepsis in vivo [15]. CLP in the mouse model of sepsis resulted in $70 \%$ mortality (Figure 5). Treatment with RX-207 and RX-205 (20 mg/kg each, $0.1 \mathrm{ml}, \mathrm{i} . \mathrm{m}), 24$ hours before surgery and once per day for the duration of the study, increased survival rate by $60 \%$ and $50 \%$, respectively. Body weight decreased to the same extent (22-23\% of starting values) in both experimental and control groups (Figure 6).

\section{Mechanism of RX-207 action}

The related quinazolinone derivatives, RX-207 and RX-205 (30 $\mu \mathrm{M})$ inhibited binding of the pro-inflammatory chemokine, MCP-3, to heparin, a highly sulfated form of the cell surface GAG, heparan sulfate (data not shown). The inhibition of MCP-3, the only one of ten heparin binding chemokines, cytokines and selectins tested, was an indication of target specificity. RX-207 and RX-205 (30 $\mu \mathrm{M})$ inhibited binding of the pro-inflammatory chemokine, IP-10, to the extracellular matrix GAG, chondroitin sulfate (CS, data not shown), extending the range of GAGs targeted by SMIGs. Spectroscopic analysis was carried out to elucidate the interaction of RX-207 with the GAGs. UV absorption spectra of RX-2007 were determined in the absence and presence of CS and heparin. The spectrum, recorded in water, exhibited multiple peaks at a wavelength greater than $200 \mathrm{~nm}$, the most intense one $\left(\varepsilon_{\max } \sim 35,000 \mathrm{M}^{-}\right.$ 
${ }^{1} \mathrm{~cm}^{-1}$ ) centered around $235 \mathrm{~nm}$ (Figure 7). Heparin induced a dose dependent reduction in the molar absorption coefficient $\left(\varepsilon_{\max }\right)$, the experimental proof of quinazolinone binding to the GAG.

At the initial phase of the titration, heparin produced a moderate (12\%) but abrupt reduction in the intensity of the main UV peak (Figure 8). Further addition of heparin induced opposite, but slower changes, restoring the molar absorption coefficient close to its original value (Figure 8). Compared with heparin, interaction of RX-207 with CS resulted in a very small decrease $(\sim 3 \%)$ of the $\varepsilon_{\max }$ value (Figure 8$)$. A small concentration of $\mathrm{NaCl}$ promptly restored the original absorption intensities of RX-207 in the presence of heparin and CS (Figure 9). No circular dichroism signals were induced either by heparin or CS (data not shown).

\section{Discussion}

Activated neutrophils have an extended half-life and an altered biochemical profile [20]. They remain in the blood stream, express inflammatory signals that alter blood vessel permeability [21] and release reactive oxygen species, proteolytic enzymes and proinflammatory cytokines [8]. Neutrophil apoptosis and phagocytosis of the apoptotic cells resolve the inflammatory response [22]. Inordinate neutrophil activity impairs innate antioxidant and anti-protease activity and stimulates indiscriminate proteolysis, the hallmark of inflammatory disorders [23]. Enteral administration of the quinazolinone related derviatives, RX-207 and RX-205, inhibited thioglycollate induced neutrophil migration (Figure 2), parenteral administration of RX-207 inhibited carrageenan induced paw edema (Figure 3) and intramuscular injection of RX-207 inhibited a neutrophil mediated increase in serum 
amylase in cerulein induced acute pancreatitis (Figure 4). RX-207 appeared to be responsible for inhibiting neutrophil recruitment and consequent inflammation in the three animal models of disease.

Intramuscular injection of RX-207 and RX-205 significantly reduced mouse mortality, 60\% and $50 \%$ respectively, in CLP-induced sepsis (Figure 5). The weights of the experimental and the control animals leveled off at $80 \%$ of starting values (Figure 6 ) and a dose of 1,500 $\mathrm{mg} / \mathrm{kg}$ (RX-205, toxicity study, not shown) did not produce any adverse clinical symptoms. The efficacy in CLP-induced sepsis, a poly-microbial sepsis that reflects clinical reality more than any other animal model of systemic inflammation [24], is predictive of therapeutic potential.

RX-207 inhibited the selective binding of MCP-3 to heparin and IP-10 binding to CS. Spectroscopic analysis of RX-207 was carried out to study its interaction with the respective GAGs (Figure 7). Heparin induced dose-dependent hypochromism [25], a reduction in $\varepsilon_{\max }$ that is experimental proof of RX-207 binding to the GAG. UV hypochromism is due to electronic interactions between the planar quinazolinone moieties of RX-207 molecules (dimers or oligomers) arranged proximally along the heparin chain. The largest reduction in the $\varepsilon_{\max }$ of RX-207 was observed around RX-207:disaccharide molar ratio of 2:1 (Figures 7 and 8), suggesting the association of two RX-207 molecules per disaccharide unit of the heparin chain. Restoration of the molar absorption coefficient to its original value by the further addition of heparin (Figure 8) might be indicative of a shift in the stoichiometry, back from 2:1 to 1:1. Due to the molar excess of heparin binding sites, the monomeric association of RX-207 became more prevalent, canceling the hypochromism. It seems that the structure of the disaccharide unit of CS (lower charge density vs. heparin, Figure 9) did not favor the 
dimeric binding of RX-207. A low concentration of $\mathrm{NaCl}$ was observed to promptly restore the original absorption intensities of RX-207 in the presence of either heparin or CS, suggesting that $\mathrm{Na}^{+}$ions effectively disrupt the Coulombic interactions between the acidic groups of GAGs and the protonated piperidine nitrogen of RX-207 (Figure 1). The dissociation of the RX-207-GAG complexes provides a rationale for the inactivity of RX-207 in inhibiting protein binding to heparin in the presence of salt.

RX207 inhibits pro-inflammatory MCP-3 binding to heparin (current manuscript) and antiinflammatory SMIGs inhibit binding of the pro-inflammatory cytokines, IL-6 and IL-8 [9]. Exactly how the anti-inflammatory SMIGs influence cytokine expression has not yet been investigated. This is a question that should be addressed given that the anti-inflammatory cytokine, IL-10, protects mice from bacterial sepsis [26] and the anti-inflammatory IL-13 prevents lethality in a mouse model of neonatal sepsis in humans, neonatal endotoxin shock [27].

The endothelial lumen of the micro-vasculature is decorated with glycocalyx, an extracellular matrix that mediates vascular barrier function, leukocyte adhesion and antioxidant defense [28]. Leukocyte adhesion molecules, embedded in the glycocalyx, are shielded from circulating leukocytes. Sepsis causes glycocalyx shedding, exposes lymphocyte adhesion molecules and liberates endogenous damage associated molecular patterns (DAMPs, [29]), including heparan sulfates, themselves inducers of sepsis [30]. Neutrophils, adhering to the glycocalyx and extravasating into proximal tissue, release heparin binding protein (HBP, [31]) that binds to cell surface proteoglycans and induces vascular leakage [32]. Spectral analysis showed that RX-207 bound directly to the GAG, heparin (Figure 7), confirmation of the mechanism of action elucidated for chemically distinct, but functionally related anti-inflammatory SMIGs [10]. Glycocalyx of the inflamed micro-vasculature 
provides the pathologic context for anti-inflammatory SMIG activity. SMIGs have the potential to prevent inflammatory neutrophils adhering to microvasculature and extravasating into proximal tissue, inhibit heparin binding proteins that cause vascular leakage and neutralize sepsis inducing heparan sulfates.

At the time of submitting this manuscript no small molecule therapy for sepsis had been approved [33]. The efficacy of SMIGs in a severe experimental model of sepsis (current report), colitis and experimental autoimmune encephalomyelitis [10], underscores the potential of the glycocalyx in general and GAGs in particular, as biological targets for the treatment of deleterious inflammation. RX-207 and RX-205 are chemically related to therapeutic quinazolinones, a number of which have anti-inflammatory activity [34], [35], [36]. Anti-inflammatory SMIGs, with defined biological targets and an elucidated mechanism of action, are potential therapeutics for diverse, but pathogenically related inflammatory disorders. 


\section{References}

1 Pavlik DJ, RW Simpson, ET Horn, L King, L Finoli. 2015. Pharmcotherapy of sepsis. Critical Care Nursing Quarterly 38: 121-36.

2 Marshall JC. 2014. Why have clinical trials in sepsis failed? Trends in Molecular Medicine 20: 195-203.

3 Fink MP and HS Warren. 2014. Strategies to improve drug development for sepsis. Nature Reviews Drug Discovery 13: 741-758.

4 Vincent JL, EO Martinez, E Silva. 2011. Evolving concepts in sepsis definitions. Critical Care Nursing Clinics of North America 23: 29-39.

5 Munford RS. 2006. Severe sepsis and septic shock: the role of gram-negative bacteremia. Annual Reviews of Pathology 1: 467-96.

6 Koh IH, JL Menchaca-Diaz, TH Koh, RL Souza, CM Shu, VE Rogerio, AM Liberatore. 2010. Microcirculatory evaluation in sepsis: a difficult task. Shock 34: (Suppl1): 27-33.

7 Wagner JG, RA Roth. 2000. Neutrophil migration mechanisms, with an emphasis on the pulmonary vasculature. Pharmacological Reviews 52: 349-374.

8 Woodfin A, MB Voisin, M Beyrau, B Colom, D Caille, FM Diapouli, GB Nash, T Chavakis, SM Albelda, GE Rainger, P Meda, BA Imhof, S Nourshargh. 2011. The junctional adhesion molecule JAM-C regulates polarized trans-endothelial migration of neutrophils in vivo. Nature Immunology 12: 761-9.

9 Harris N, F Yurgenzon Kogan, G II'kova, S Juhas, O Lahmy, YI Gregor, J Koppel, R Zhuk, P Gregor. 2014. Small molecule inhibitors of protein interaction with glycosaminoglycans 
(SMIGs), a novel class of bioactive agents with anti-inflammatory properties. Biochimica et Biophysica Acta 1840: 245-254.

10 Harris N, J Koppel, F Zsila, S Juhas, G II'kova, F Yurgenzon Kogan, O Lahmy O, G Wildbaum, N Karin, R Zhuk, P Gregor. 2016. Mechanism of action and efficacy of RX-111, a thieno[2,3-c]pyridine derivative and small molecule inhibitor of protein interaction with glycosaminoglycans (SMIGs), in delayed-type hypersensitivity, TNBS-induced colitis and experimental autoimmune encephalomyelitis. Inflammation Research 65: 285-294.

11 Xie X, AS Rivier, A Zakrzewicz, M Bernimoulin, X-L Zeng, HP Wesseli, M Schapira, O Spertini. 2000. Inhibition of Selectin-mediated Cell Adhesion and Prevention of Acute Inflammation by Nonanticoagulant Sulfated Saccharides. J. Biological Chemistry 275: 34818-34825.

12 Torres SR, TS Fröde, GM Nardi, N Vita, R Reeb, P Ferrara, RM Ribeiro-do-Valle, RC Farges. 2000. Anti-inflammatory effects of peripheral benzodiazepine receptor ligands in two mouse models of inflammation. European Journal of Pharmacology 408: 199-211.

13 Cuzzocrea S, E Mazzon, L Dugo, I Serraino, T Centorrino, A Ciccolo, FA Van de Loo, D Britti, AP Caputi, C Thiemermann. 2002. Inducible nitric oxide synthase-deficient mice exhibit resistance to the acute pancreatitis induced by cerulein. Shock 17: 416-22.

14 Steinberg WM, SS Goldstein, ND Davis, J Shamma'a, K Anderson. 1985 Diagnostic Assays in Acute Pancreatitis: A Study of Sensitivity and Specificity. Annals of Internal Medicine 102: $576-80$.

15 Fink MP and SO Heard. 1990. Laboratory Models of Sepsis and Septic Shock. Journal of Surgical Research 49: 186-196. 
16 Henderson RB, JAR Hobbs, M Mathies, N Hogg. 2003. Rapid recruitment of inflammatory monocytes is independent of neutrophil migration. BLOOD 102: 328-335.

17 Kipari T, S Watson, K Houlberg, S Lepage, J Hughes, JF Cailhier. 2009. Lymphocytes modulate peritoneal leukocyte recruitment in peritonitis. Inflammation Research 58: 55360.

18 Vinegar R, JF Truax, JL Selph, PR Johnston, AL Venable, KK McKenzie. 1987. Pathway to carrageenan-induced inflammation in the hind limb of the rat. Fed Proc 46:118-26.

19 Niederau C, LD Ferrell, JH Grendell. 1985. Cerulein induced acute necrotizing pancreatitis in mice: protective effects of proglumide, benzotript, and secretin. Gastroenterology 88: $1192-1204$.

20 Fournier BM, CA Parkos. 2012. The role of neutrophils during intestinal inflammation. Mucosal Immunology 5: 354-66.

21 Kolaczkowska E, P Kubes. 2013. Neutrophil recruitment and function in health and inflammation. Nature Reviews Immunology 13: 159-75.

22 Nauseef WM, N Borregaard. 2014. Neutrophils at work. Nature Immunology 15: 602-11.

23 Mantovani A, MA Cassatella, C Costantini, S Jaillon. 2011. Neutrophils in the activation and regulation of innate and adaptive immunity. Nature Reviews Immunology 11: 51931.

24 Siempos II, HC La, Y Ding, ME Choi, AMK Choi, SW Ryter. 2014. Cecal Ligation and Puncture-induced Sepsis as a Model To Study Autophagy in Mice. Journal of Visualized Experiments 84 e51066: 1-7. 
25 Zsila F. 2015. Glycosaminoglycans are potential pharmacological targets for classic DNA minor groove binder drugs berenil and pentamidine. Physical Chemistry Chemical Physics $17: 24560-5$.

26 Howard M, Muchamuel T, Andrade S, Menon S. 1993. Interleukin 10 protects mice from lethal endotoxemia. Journal of Experimental Medicine 177(4):1205-8.

27 Nicoletti F, G Mancuso, V Cusumano, R Di Marco, P Zaccone, K Bendtzen, G Teti. 1997. Prevention of endotoxin-induced lethality in neonatal mice by interleukin-13. European Journal of Immunology 27(6):1580-3.

28 Martin L, P Koczera, E Zechendorf, T Schuerholz. 2016. The Endothelial Glycocalyx: New Diagnostic and Therapeutic Approaches in Sepsis. BioMed Research International 2016: 1-8.

29 Huang ML, CJ Fisher, K Godula. 2016. Glycomaterials for probing host-pathogen interactions and the immune response. Experimental Biology and Medicine 241: 10421053.

30 Martin L, C Peters, S Schmitz, J Moellmann, A Martincuks, N Heussen, M Lehrke, G Müller-Newen, G Marx, T Schuerholz. 2015. Soluble heparan sulfate in serum of septic shock patients induces mitochondrial dysfunction in murine cardiomyocytes. Shock 44: 569-577.

31 Bentzer P, J Fisher, HJ Kong, M Mörgelin, JH Boyd, KR Walley, JA Russell, A Linder. 2016. Heparin-binding protein is important for vascular leak in sepsis. Intensive Care Medicine Experimental 4:1-16.

32 Gautam N, AM Olofsson, H Herwald, LF Iversen, E Lundgren-Akerlund, P Hedqvist, 
KE Arfors, H Flodgaard, L Lindbom. 2001. Heparin-binding protein (HBP/CAP37): a

missing link in neutrophil-evoked alteration of vascular Permeability. Nature Medicine 7:

$1123-1127$.

33 Gotts JE, MA Matthay. 2016. Sepsis: pathophysiology and clinical management. British Medical Journal 353: 1585-1605.

34 Chatterjee N, S Da, D Bose, S Banerjee, S Das, D Chattopadhyay, KD Saha. 2012. Exploring the anti-inflammatory activity of a novel 2-phenylquinazoline analog with protection against inflammatory injury. Toxicology and Applied Pharmacology 264: 182-191.

35 Pacheco de Oliveira MT, TR de Oliveira Ramalho, LK Paiva Ferreira, AL Araújo Lima, M Barbosa Cordeiro, H Ferreira Costa, LC Rodrigues, MR Piuvezam. 2015. Synthesis, toxicity study and anti-inflammatory effect of MHTP, a new tetrahydroisoquinoline alkaloid. Immunopharmacology Immunotoxicologyl 37: 400-12.

36 Vellinga NAR, G Veenstra, C Scorcella, M Koopmans, EN van Roon, C Ince, EC Boerma. 2015. Effects of ketanserin on microcirculatory alterations in septic shock: An open-label pilot study. Journal of Critical Care 30: 1156-1162. 
<smiles>CC(C)N1CCC(NC(=O)c2ccc3c(=O)n4c(nc3c2)CCCCC4)CC1</smiles>

A<smiles>CN1CCC(N(C)C(=O)c2ccc3c(=O)n4c(nc3c2)CCC4)CC1</smiles>

B

Fig. 1 (A) RX-207, (B) RX-205

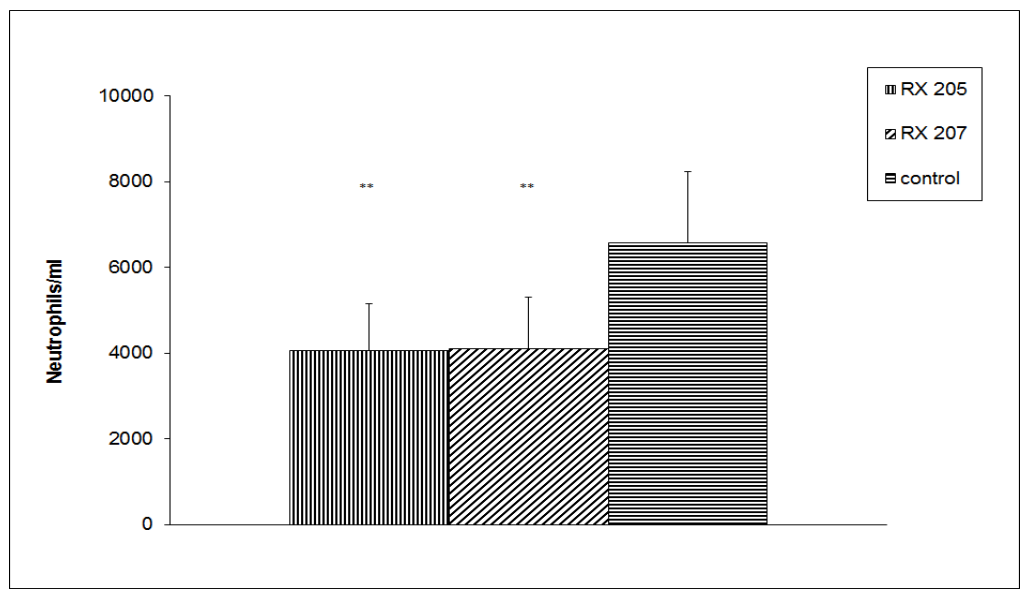

Fig. 2 Inhibition of neutrophil migration by RX-205 and RX-207 (50 mg/kg, per $o s)$ in a mouse model of thioglycollate induced peritonitis $(* * p<0.01)$ 
Fig. 3 Anti-inflammatory activity of RX-207 in a mouse model of carrageenan-induced edema. RX-207 (1 mg/kg) was administered i.m. one hour before injecting carrageenan. Paw edema was determined 4 hours after administering carrageenan. The $Y$ axis represents difference in paw thickness between carrageenan treated and non-treated paws $(* * * p<0.001)$

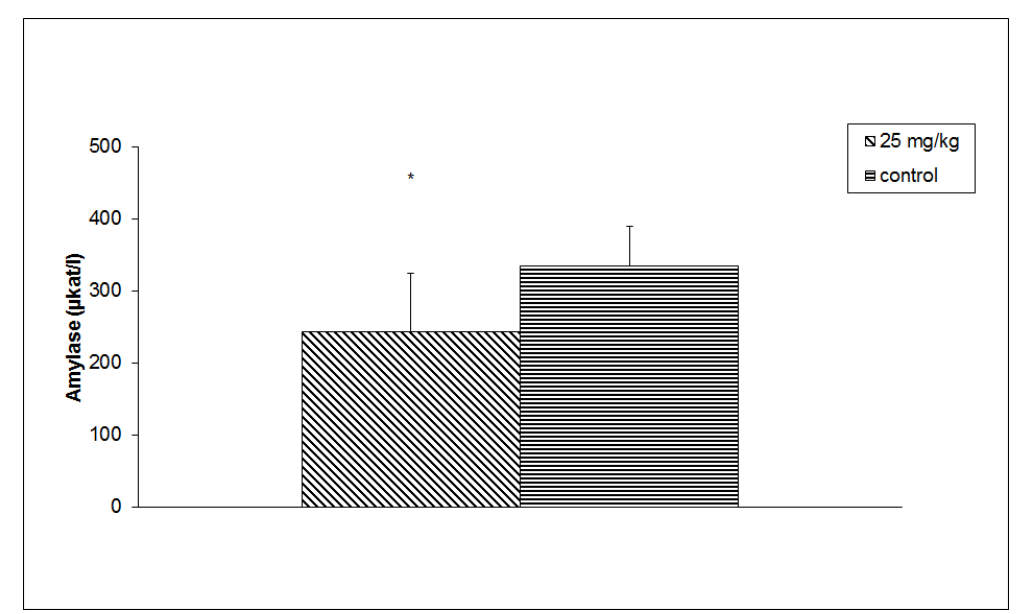

Fig. 4 Therapeutic activity of RX-207 in a mouse model of acute pancreatitis. RX-207 $(25 \mathrm{mg} / \mathrm{kg}$ ) was administered i.m. one hour before injecting cerulein. Serum amylase was analyzed one hour after the last injection of cerulein $\left({ }^{*} p<0.05\right)$ 


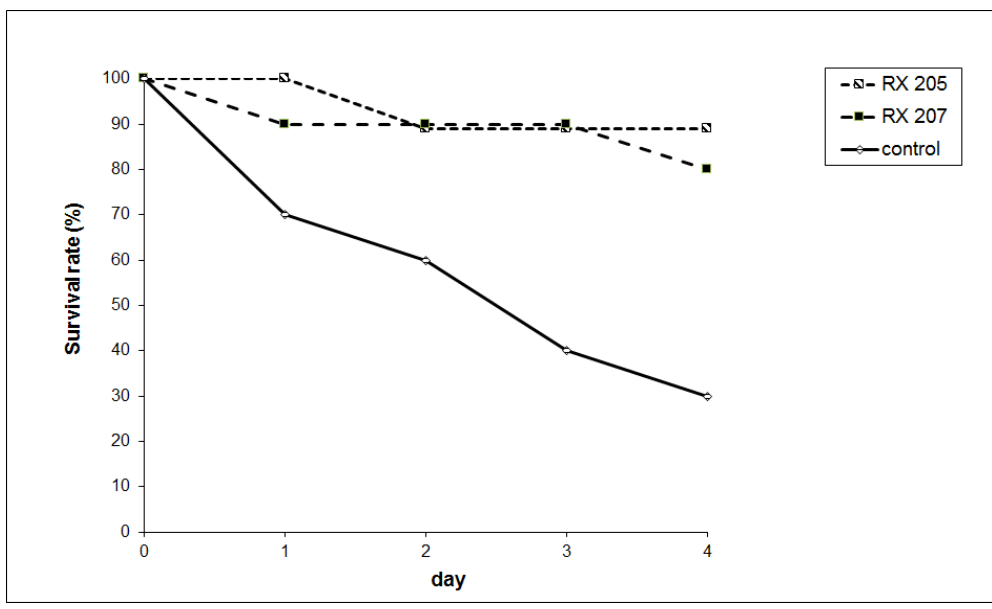

Fig. 5 Therapeutic activity (survival rate) of RX-205 $(20 \mathrm{mg} / \mathrm{kg}$, i.m., $\left.{ }^{*} p=0.0123\right)$ and RX-207 (20 mg/kg, i.m., *p=0.0233) in the mouse model of CLP-induced sepsis. *Log-rank (MantelCox) test for CLP survival

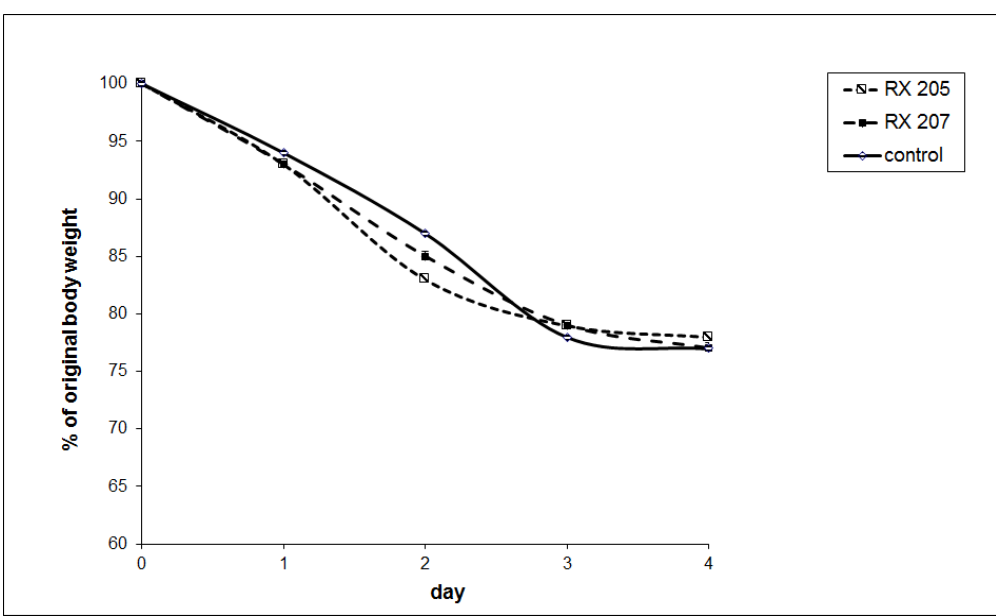

Fig. 6 Change in body weight of mice treated with RX-205 $(20 \mathrm{mg} / \mathrm{kg}$, i.m.) and RX-207 (20 mg/kg, i.m.) in the mouse model of CLP-induced sepsis 


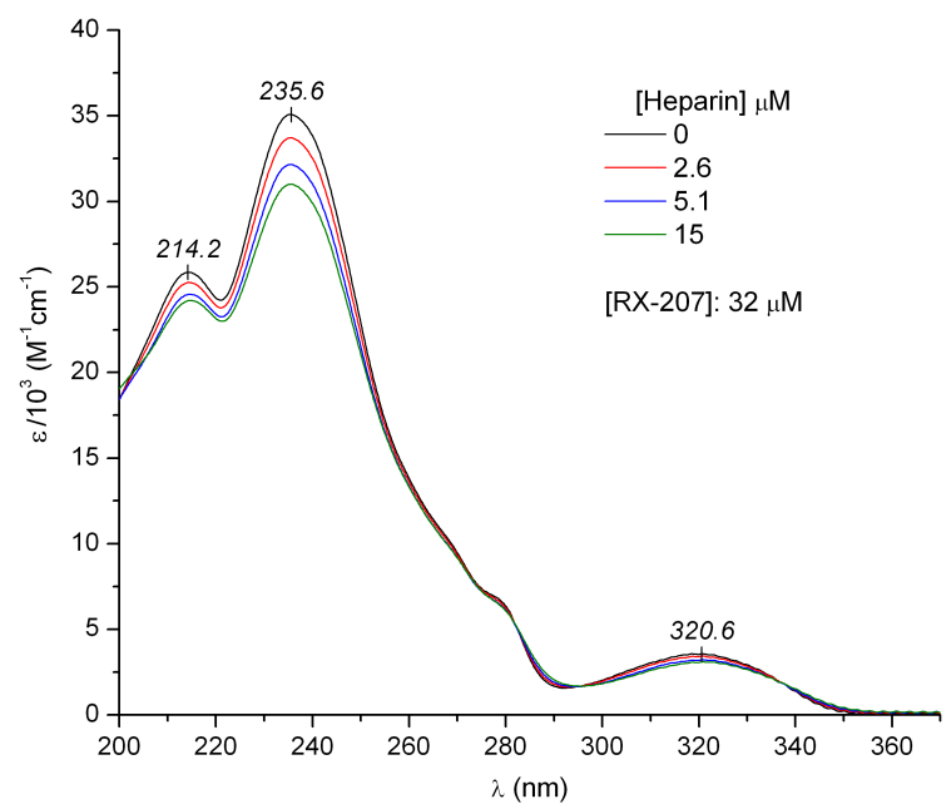

Fig. 7 Effect of heparin on the UV absorption spectrum of RX-207 measured in aqueous solution

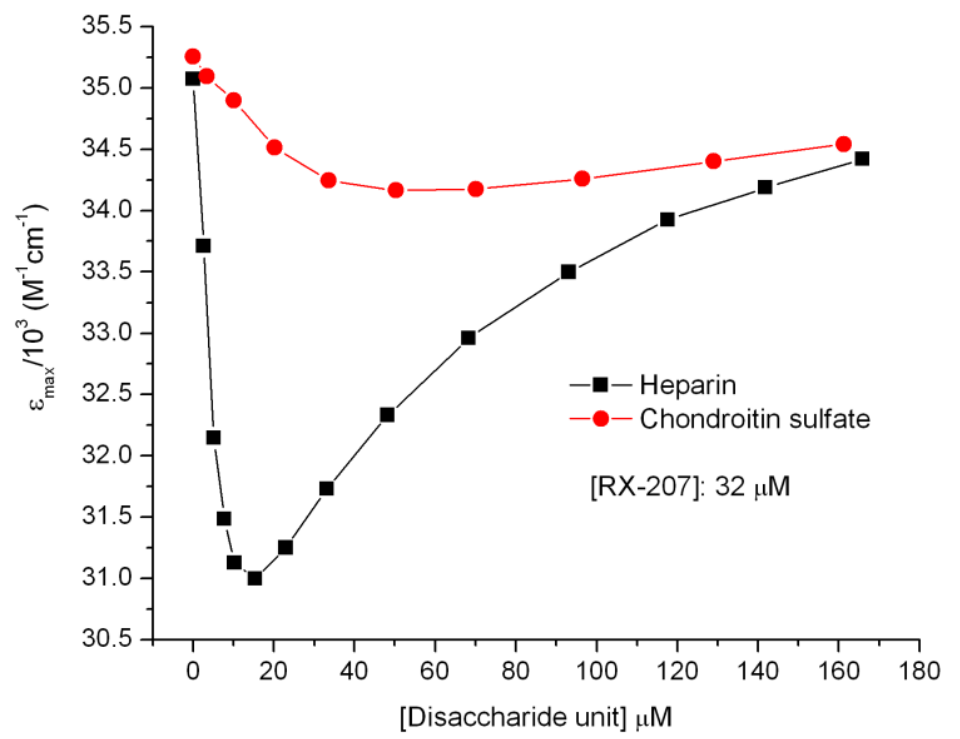

Fig. 8 Effect of addition of heparin and CS on the $\varepsilon_{\max }$ value of RX-207 
1

2

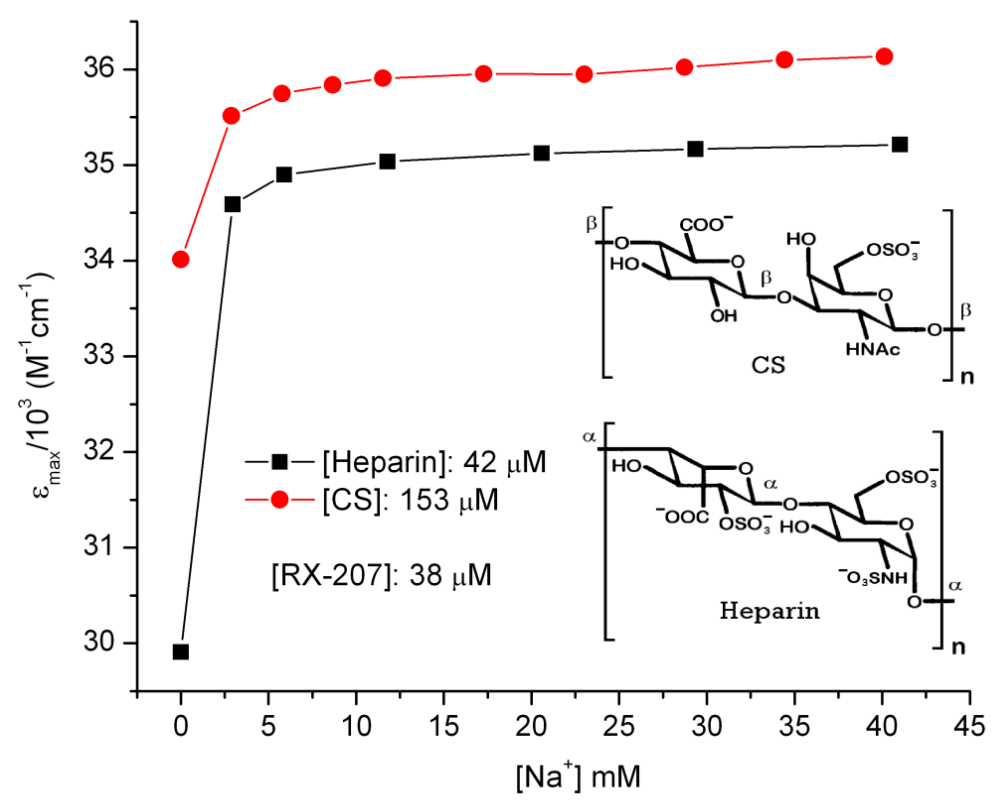

Fig. 9 Effect of sodium chloride on the $\varepsilon_{\max }$ of RX-207 bound to heparin and CS. Inset shows the average repeating disaccharide unit of CS and heparin 